\title{
Peptide vaccine immunotherapy biomarkers and response patterns in pediatric gliomas
}

\author{
Sören Müller, ${ }^{1}$ Sameer Agnihotri, ${ }^{2}$ Karsen E. Shoger, ${ }^{2}$ Max I. Myers, ${ }^{2}$ Nicholas Smith, ${ }^{3}$ \\ Srilakshmi Chaparala, ${ }^{4}$ Clarence R. Villanueva, ${ }^{2}$ Ansuman Chattopadhyay, ${ }^{4}$ Adrian V. Lee, ${ }^{3}$ \\ Lisa H. Butterfield, ${ }^{5}$ Aaron Diaz, ${ }^{1}$ Hideho Okada, ${ }^{1}$ Ian F. Pollack, ${ }^{2}$ and Gary Kohanbash ${ }^{2}$ \\ 'Department of Neurological Surgery, University of California, San Francisco, San Francisco, California, USA \\ ${ }^{2}$ Department of Neurological Surgery, ${ }^{3}$ Department of Pharmacology, ${ }^{4}$ Health Sciences Library System, and \\ ${ }^{5}$ Departments of Medicine, Surgery, and Immunology and Clinical and Translational Science Institute, University of \\ Pittsburgh, Pittsburgh, Pennsylvania, USA.
}

\begin{abstract}
Low-grade gliomas (LGGs) are the most common brain tumor affecting children. We recently reported an early phase clinical trial of a peptide-based vaccine, which elicited consistent antigenspecific T cell responses in pediatric LCG patients. Additionally, we observed radiologic responses of stable disease (SD), partial response (PR), and near-complete/complete response (CR) following therapy. To identify biomarkers of clinical response in peripheral blood, we performed RNA sequencing on PBMC samples collected at multiple time points. Patients who showed CR demonstrated elevated levels of $\mathrm{T}$ cell activation markers, accompanied by a cytotoxic $\mathrm{T}$ cell response shortly after treatment initiation. At week 34, patients with CR demonstrated both IFN signaling and Poly-IC:LC adjuvant response patterns. Patients with PR demonstrated a unique, late monocyte response signature. Interestingly, HLA-V expression, before or during therapy, and an early monocytic hematopoietic response were strongly associated with SD. Finally, low IDO1 and $P D-L 1$ expression before treatment and early elevated levels of T cell activation markers were associated with prolonged progression-free survival. Overall, our data support the presence of unique peripheral immune patterns in LGG patients associated with different radiographic responses to our peptide vaccine immunotherapy. Future clinical trials, including our ongoing phase II LGG vaccine immunotherapy, should monitor these response patterns.
\end{abstract}

Authorship note: SM and SA contributed equally to this work. $A D$, HO, IFP, and GK are co-senior authors.

Conflict of interest: $\mathrm{HO}$ is an inventor on US patent application no. 60,611,797 (utility patent application), "Identification of An IL-13 Receptor Alpha2 Peptide Analogue Capable of Enhancing Stimulation of CliomaSpecific CTL Response." An exclusive licensing agreement has been completed on this application between the University of Pittsburgh and Stemline Inc. Due to the potential conflicts of interest, $\mathrm{HO}$ did not solely interpret any data in the current study.

Submitted: December 6, 2017 Accepted: February 28, 2018 Published: April 5, 2018

\section{Reference information:} JCI Insight. 2018;3(7):e98791. https:// doi.org/10.1172/jci.insight.98791

\section{Introduction}

Numerous studies evaluating peptide-based vaccine approaches for adult and pediatric brain tumors have demonstrated the feasibility and safety associated with this approach (1-6). Despite frequently eliciting biologic responses, tumor shrinkage is often not observed. Multiple factors likely contribute to this, including antigen loss (6), low MHC expression on tumor cells (7), inhibitory checkpoint pathways (8), and the accumulation of immunosuppressive T regulatory and myeloid cells (9).

Type 1-polarized T cells expressing IFN can migrate to and mediate effective antiglioma immunity (10, 11); however, tumor-associated myeloid cells can produce immunosuppressive cytokines, altering these $\mathrm{T}$ cells to an ineffective type 2-polarized state. Accordingly, we recently found that the presence of tumor-infiltrating bone marrow-derived myeloid cells is associated with decreased overall survival in patients with low-grade glioma (LGG) (12). Additionally, we have demonstrated that the COX-2 pathway and prostaglandin-E2 (PGE2) are critical for the immunosuppressive function of myeloid-derived suppressor cells in glioma (13). The changing tumor microenvironment and peripheral immune environment during vaccine therapy may effect these immunosuppressive populations and must therefore be evaluated as mechanisms of resistance in glioma immunotherapy.

We recently reported on a peptide vaccine immunotherapy trial for children with LGG (14). Patients were vaccinated with immunogenic peptides targeting 3 HLA-A*0201-restricted glioma-associated antigen epitopes (EphA2 ${ }_{883-891}, \mathrm{IL}-13 \mathrm{Ra} 2_{345-353}$, and survivin $_{96-104}$ ) emulsified in Montanide-ISA-51, in combination with a TLR3 agonist and polyinosinic-polycytidylic acid stabilized by 
Table 1. Summary of metadata for patients included in this study

\begin{tabular}{cccc}
\hline Patient no. & Best response & PFS (mo.) & Samples drawn at weeks \\
1 & SD & 16.1 & $0,6,15,34,70$ \\
2 & SD & 20.8 & $0,6,15,34,70$ \\
3 & SD & 6.7 & $0,6,15$ \\
4 & Near-CR & 69.3 & $0,6,15,34,70,86$ \\
5 & CR & 57.7 & $0,6,15,34,70,86$ \\
6 & SD & 9.8 & $0,6,15,34$ \\
7 & MR/PR transient & 9.9 & $0,6,15,34$ \\
8 & SD & 7.9 & $0,6,15,34$ \\
9 & PR & 43.2 & $0,6,15,34,70,86$ \\
10 & SD & 7.5 & $0,6,15,34$ \\
11 & SD & 3.4 & $0,6,15$ \\
12 & PR & 10.9 & $0,6,15,34$
\end{tabular}

$\mathrm{CR}$, complete response; MR, minor response; PFS, progression-free survival; PR, partial response; SD, stable disease.

lysine and carboxymethylcellulose (Poly-IC:LC) as an adjuvant. Vaccines were administered in 3-week intervals for 8 courses of treatment, followed by additional rounds of treatment at 6 -week intervals for up to 2 years. Remarkably, in all 12 evaluable patients, we detected a biologic response to at least one peptide by enzyme-linked immunosorbent spot (ELISPOT) analysis (14). Furthermore, of these 12 patients, 7 experienced best responses of stable disease (SD), 3 experienced transient-partial response/ partial response (PR), and 2 experienced near-complete response/complete-response (CR). Mean progression-free survival (PFS) for patients with $\mathrm{CR}$ was $>72.85( \pm 8.70)$ months, for patients with PR was $24.72( \pm 24.75)$ months, and $10.34( \pm 6.00)$ months for SD patients, with durable responses in both patients with $\mathrm{CR}$ and 1 of the patient with PR (Table 1). These results led us to develop a phase II study using this approach (NCT02358187).

To identify biomarkers of response and peripheral immunoreactivity patterns, we performed RNA sequencing (RNA-seq) on peripheral blood mononuclear cells (PBMCs) from patients prior to the start of vaccine treatment and at multiple time points during treatment ( $n=54$ total samples). We found that $H L A-V$, a class I MHC pseudogene, and GSTM1 expression could differentiate between SD, PR, and CR patients in our trial. Furthermore, T cell activity genes (GZMB and $P R F 1$ ) were elevated at an early time point in CR patients compared with SD and PR patients, whereas monocytic pathways were elevated at early time points in SD (week 6) and PR (week 15) patients. Significantly, at week 34 following the start of treatment, only CR patients demonstrated strong induction of both IFN signaling and Poly-IC response pathways. Furthermore, genes involved in DC activation of T cells were correlated with ELISPOT responses to survivin. Finally, low expression levels of genes encoding immune checkpoint proteins at week 0 and high expression levels of T cell activation markers at week 6 were associated with longer PFS. Overall, our data suggest the presence of unique peripheral immune pathways associated with pediatric LGG patient response to peptide vaccine immunotherapy. Although intriguing, based on the small sample size, our identified gene signatures will ultimately need to be validated in prospective randomized trials. Finally, our findings highlight the utility of RNA-seq as a broadly useful tool for the monitoring of peripheral immune responses in patients receiving immunotherapies.

\section{Results}

Unsupervised analysis reveals unique gene expression clustering of $S D, P R$, and $C R$ patients. PBMCs were collected from LGG patients at multiple time points relative to the initial peptide vaccine treatment, including week 0 (pretreatment), 6, and 15 and when available week 34, 70, and 86 (Figure 1A and Table 1). Subsequently, we performed RNA-seq on each PBMC sample and generated a total of approximately 2 billion reads. Each library consisted of approximately 36,000,000 reads $(35,955,102 \pm 4,404,110)$ that were uniquely assigned to the human transcripts in each sample, robustly measuring the expression of 36,410 coding and noncoding genes. For each sample, expression values were scaled to transcripts per kilobase million (TPM). 
A

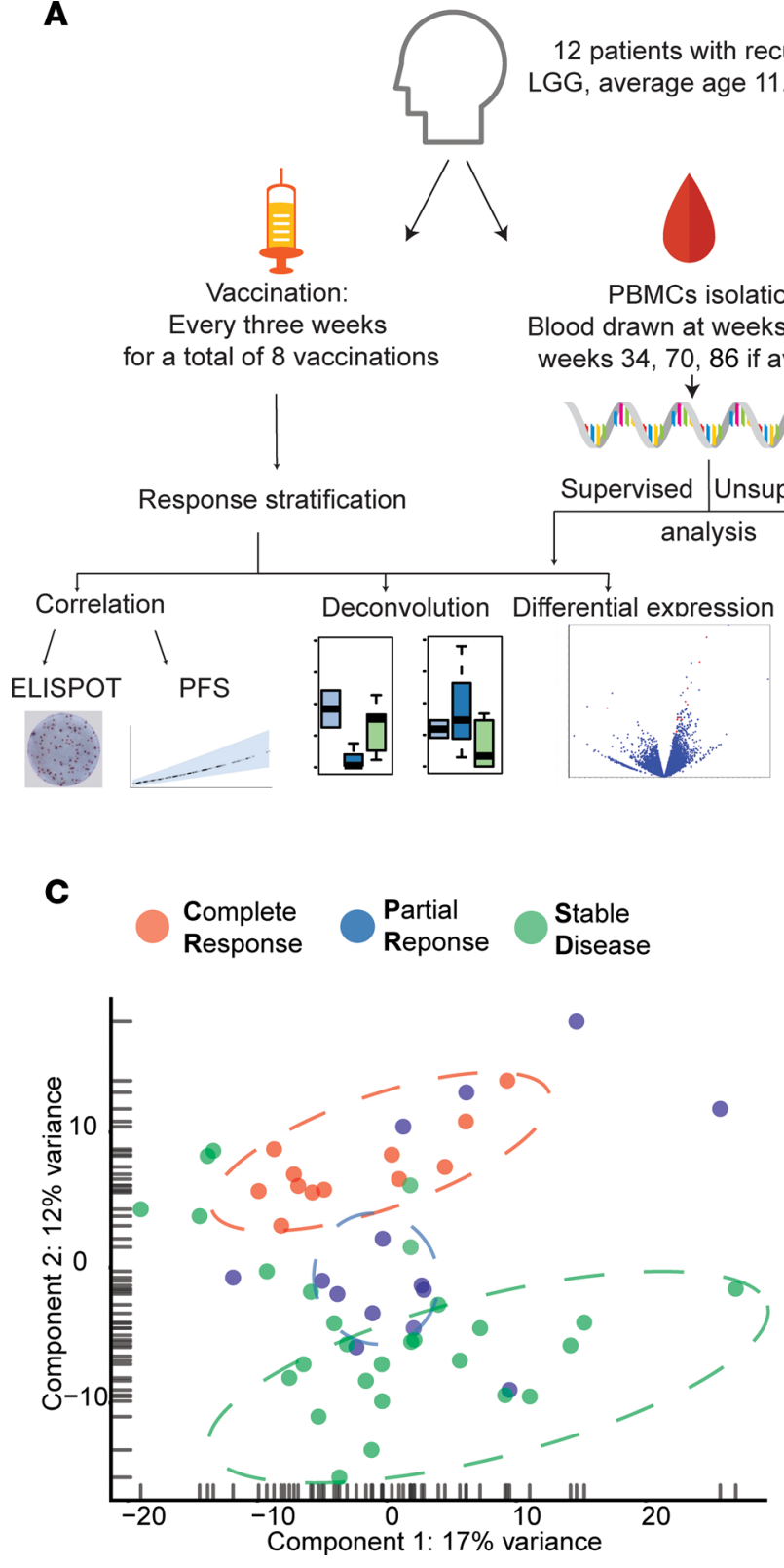

B

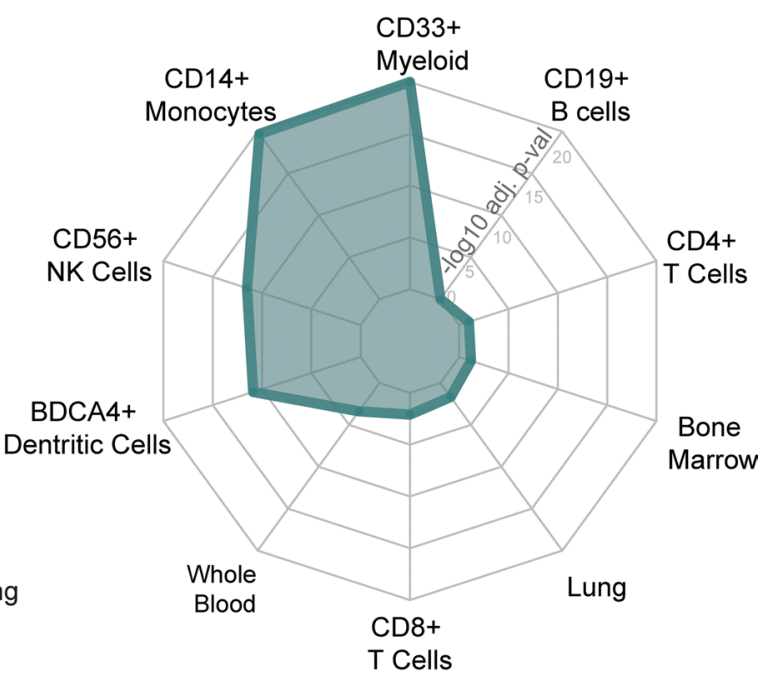

Figure 1. Unsupervised clustering of PMBC RNA-seq profiles reveals grouping by treatment response. (A) Overview of the study design and experimental procedure. PFS, progression-free survival. (B) Estimate of cell types enriched in sequenced PBMCs ( $n=12$ patients) based on the 300 most highly expressed genes across all samples. The adjusted enrichment $P$ value (1-tailed Fisher's exact test, Benjamini-Hochberg correction) is indicated by the bold green line on the web. The respective cell population is given outside of the web. (C) Unbiased PCA of PBMC samples ( $n=12$ patients, 54 total samples). Treatment response is indicated by color; the explained variance by each PC is given below the axes. (D) Consensus clustering of PBMC samples. Treatment response is indicated by color (top); time from first treatment is indicated in green on the right.

To assess data quality and reliability, we used Cten (15) to identify enriched cell types after averaging gene expression across all 54 samples. As expected, samples were significantly enriched in $\mathrm{T}$ cells, B cells, macrophages, DCs, and other immune cell populations (Figure 1B). Next, we performed principal component analysis, an unsupervised dimensionality reduction method, in the space of all 36,410 genes. Samples from patients with best responses of CR, PR, or SD generally clustered independently from other response groups (Figure 1C). Unsupervised consensus clustering (16) similarly revealed groups consisting mainly of PBMC samples from patients with the same radiologic outcome (Figure 1D). Notably, we did not observe any unique clustering of samples based on the time after initiation of treatment in PCA (data not shown) or consensus clustering (Figure 1D). These data 
A

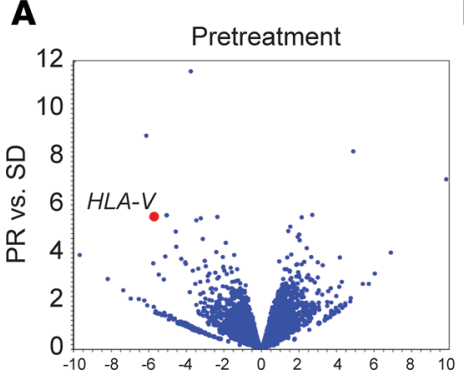

$\log _{2}$ fold change

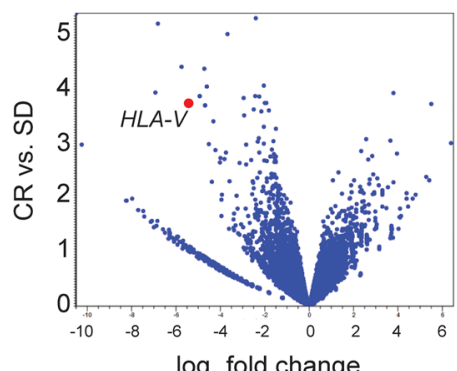

$\log _{2}$ fold change

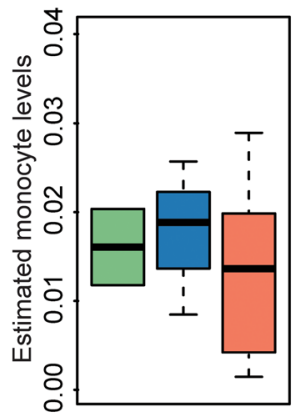

E

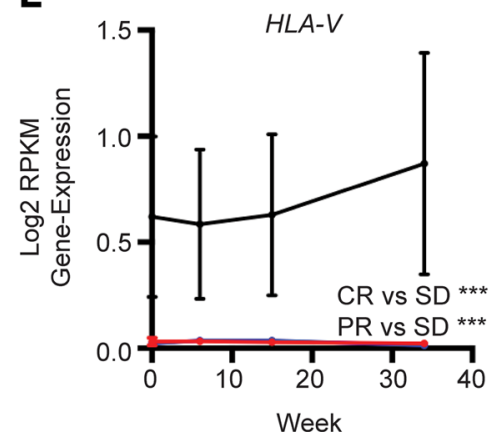

B

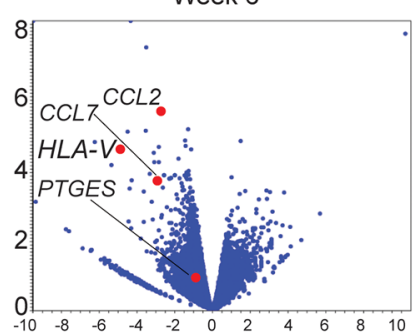

$\log _{2}$ fold change

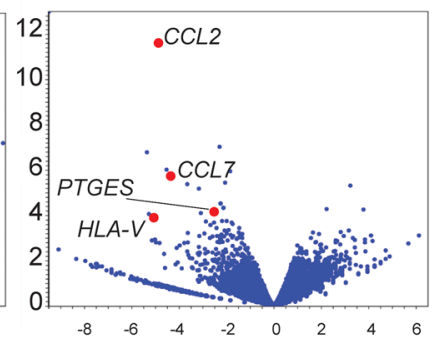

$\log _{2}$ fold change

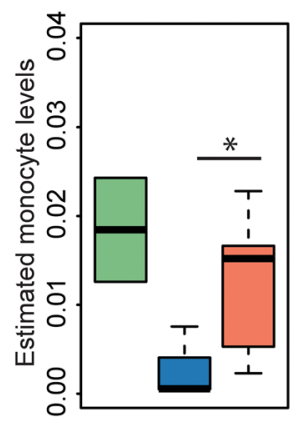

$\mathbf{F}$

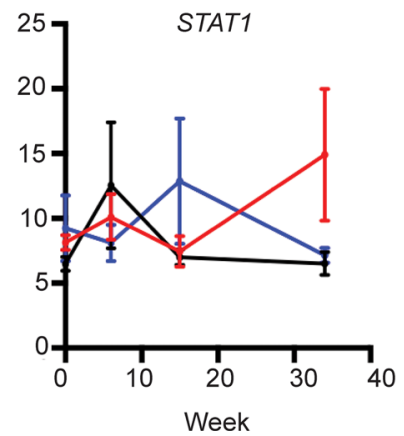

C

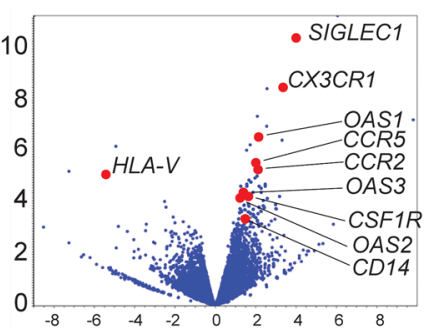

$\log _{2}$ fold change

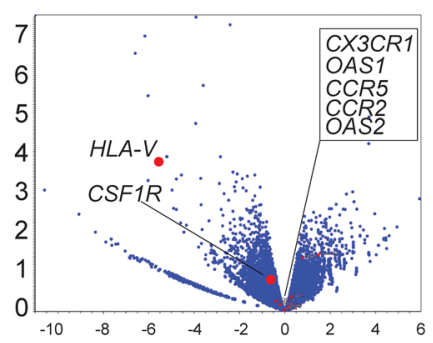

$\log _{2}$ fold change

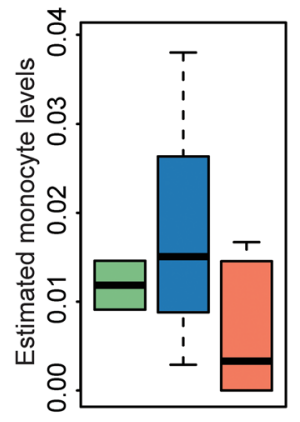

G

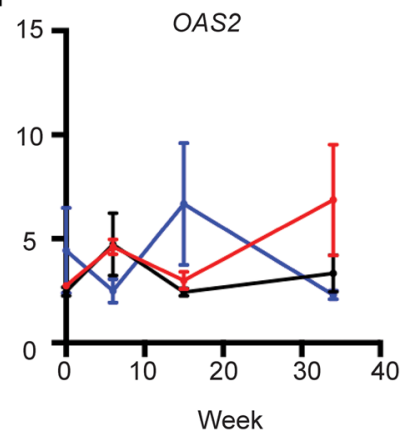

Week 34

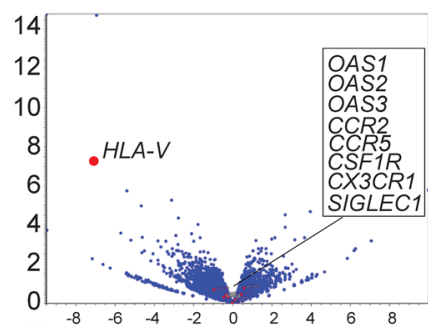

$\log _{2}$ fold change

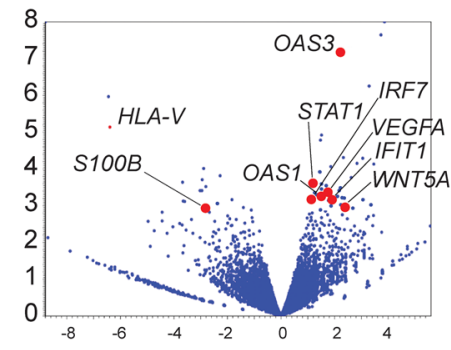

$\log _{2}$ fold change

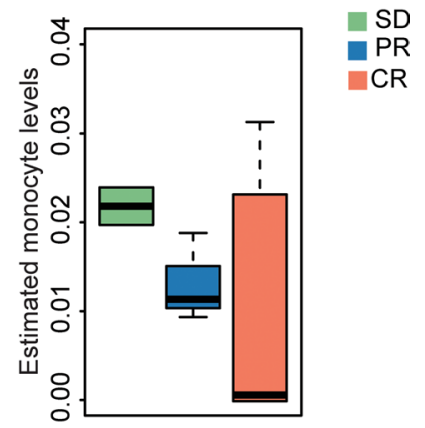

Figure 2. Differential expression analysis reveals several immune-related genes associated with response at various time points. (A-D) Volcano plots comparing fold change ( $x$ axis) and $-\log _{10}(P$ value) ( $y$ axis, edgeR) for 36,410 expressed genes at weeks $0,6,15$, and 34 between PR ( $n=3$ patients) and SD ( $n=$ 7) and CR $(n=2)$ and SD $(n=7)$ patients. Specific immune-related genes are highlighted in red. Relative estimates of the monocyte abundance in each group (color) at each time point were calculated using xCell. *Adjusted $P<0.05$ ( $t$ test, Bonferroni correction). (E-G) Average ( \pm SD) expression of HLA-V, STAT1, and OAS1 ( $y$ axis) from week 0 to 34 ( $x$ axis). Response groups are indicated by color. ${ }^{* *}$ Adjusted $P<0.0001$ (Tukey's test with multiple testing correction).

suggest that transcriptomic analysis of PBMCs allows the prediction of patient vaccine response in an unsupervised manner.

Differential expression analysis reveals distinct peripheral responses correlating with response to peptide vaccine. To evaluate the differential expression of specific genes, we next compared SD versus PR patients $(n=7$ and $n=$ 3 , respectively) and SD versus CR patients ( $n=7$ and $n=2$, respectively). We performed analysis independently at each time point (Figure 2, A-D, and Supplemental Figure 1; supplemental material available online with this article; https://doi.org/10.1172/jci.insight.98791DS1) and identified several immune-related genes that 
A
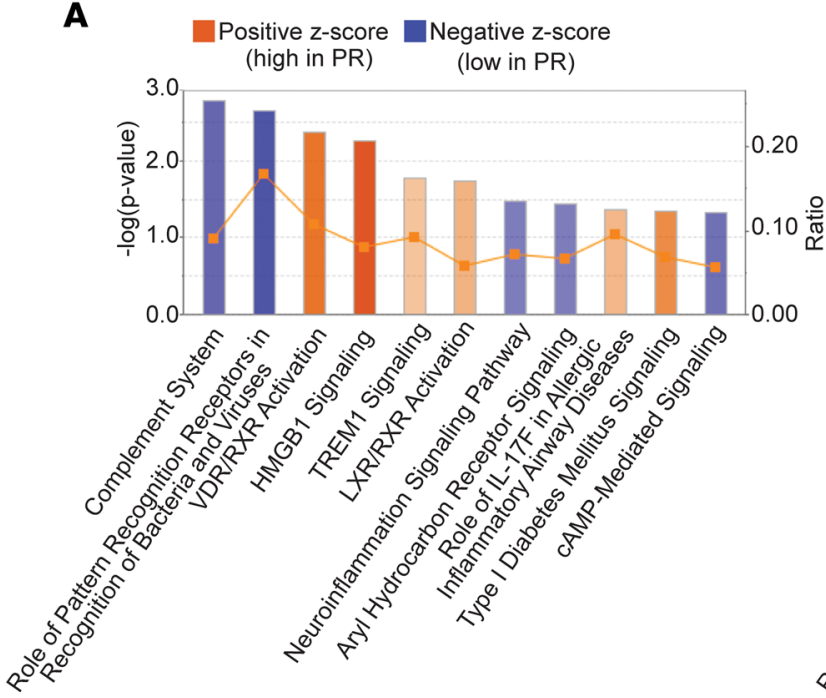

B
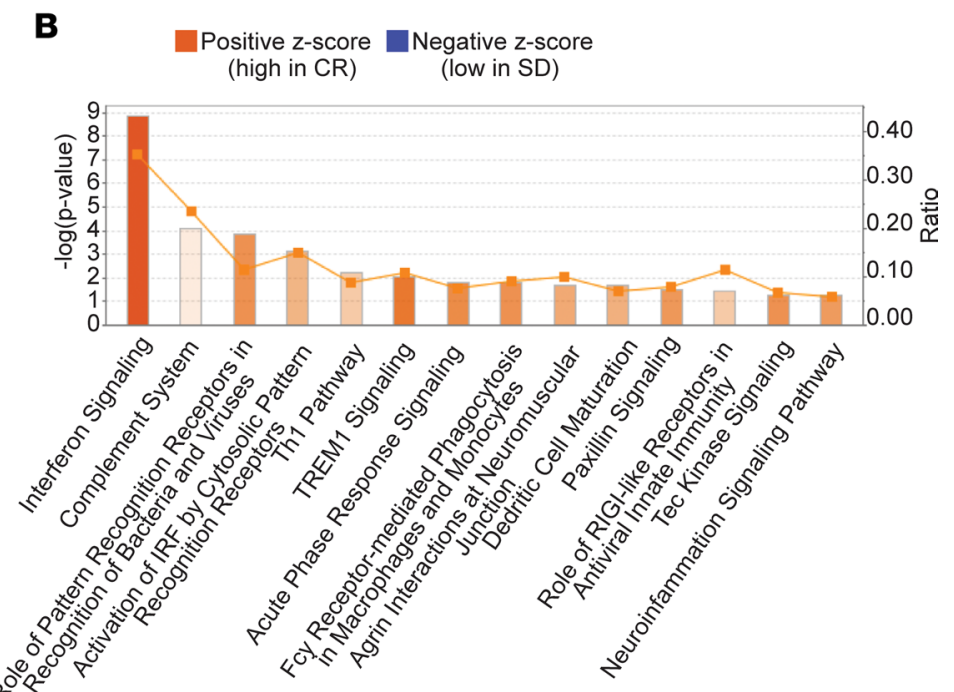

Figure 3. Induced and repressed pathways associated with treatment response at different time points. (A) Significance ( $y$ axis, Fisher's exact test) of pathway enrichment for genes differentially expressed between PR and SD patients at week 34 . The ratio of differentially expressed genes to the total number of genes in the pathway is shown by the orange line. The average expression change of genes in each pathway between PR and SD patients is indicated by color (Z-score). (B) As in A, for genes differentially expressed between CR and SD patients.

stood out as having significant differential expression (computed via edgeR, ref. 17) between groups. Interestingly, at both pretreatment and every other time point evaluated, $H L A-V$ expression was increased in SD versus CR/PR patients (Figure 2, A-E). At week 6 following the initial treatment, we observed higher levels of chemokines promoting monocytic hematopoiesis (CCL2 and CCL7) and PTGES, which regulates PGE2 synthesis, in patients with SD compared with PR and CR patients (Figure 2B). At week 15 following the initial treatment, PR patients, but not CR patients, displayed elevated levels of genes related to a monocyte response compared with SD patients (Figure 2C). At week 34 following initial treatment, CR patients, but not PR patients developed significantly elevated IFN responses (STAT1 and OAS2) compared with SD patients. (Figure 2D). We next used xCell (18) to estimate the fraction of different cell types in each PBMC sample based on the expression levels of known, cell-type specific markers. Estimates of this "deconvolution" method have been shown to be strongly correlated with true fractions of different cell types obtained via FACS sorting (18). In line with our previous observations, at 6 weeks SD patients exhibited the highest monocyte levels. Furthermore, monocyte levels were significantly higher in CR patients versus PR patients (Figure 2B). This changed after 15 weeks, at which point PR patients showed elevated monocyte levels compared with CR patients (Figure 2C). At week 34, monocyte levels remained low in CR patients (Figure 2D).

Interestingly, we observed that STAT1 transcripts, a marker of effective immune responses, were increasing in CR patients from weeks 0-34. Although comparable to CR patient levels from weeks 0-15, PR patient STAT1 transcripts significantly declined by week 34 (Figure $2 \mathrm{~F}, P<0.05$ at week 34 ). OAS2, another positive marker of an immune response, followed a similar trend to STAT1 (Figure 2G).

In order to identify biological processes altered between patients dichotomized as CR versus SD, we performed gene set enrichment analysis (GSEA) at week 0 (before vaccination) to identify any blood biomarkers or pathways that may indicate a response to our vaccine. Compared with CR patients, SD patients at week 0 showed a significant enrichment of genes in several immune pathways that could indicate a poor immune response: reduction in effector T cells expressing miR-155 (Supplemental Figure 2A, $P=0.02$ ) and memory $\mathrm{T}$ cell gene sets compared with $\mathrm{CR}$ patient PBMCs (Supplemental Figure $2 \mathrm{~B}, P$ $=0.018$ ). Additionally, SD patients showed an enrichment of genes associated with type 1 diabetes compared with CR patients (Supplemental Figure 2C, $P=0.035$ ). miR-155 is known to be induced by TLR3 agonists such as Poly-IC $(19,20)$, suggesting that responsiveness to the adjuvant may be important for clinical responses. Additionally, miR-155 is known to be required for effector $\mathrm{CD}^{+} \mathrm{T}$ cell responses in cancer (21). Furthermore, we compared PR patients to SD patients at week 0 and found that SD patients showed an enriched pathway signature associated with immune genes upregulated in acute respiratory syncytial virus infection (Supplemental Figure 2D, $P<0.001$ ). 
Table 2. Predicted upstream regulators of gene pathways at week 34 after initial treatment

\begin{tabular}{|c|c|c|c|}
\hline \multicolumn{4}{|c|}{ Best response, week 34} \\
\hline \multicolumn{2}{|c|}{ PR vs. SD } & \multicolumn{2}{|r|}{ CR vs. SD } \\
\hline Activated & Inhibited & Activated & Inhibited \\
\hline Poly rl:rC-RNA & KCNK9 & IFNA2 & ASXL1 \\
\hline Nitrofurantoin & IGHM & IFNG & ZNF106 \\
\hline IL-12 (complex) & NFIL3 & IRF7 & NT5E \\
\hline $\begin{array}{l}\text { 5-0-mycolyl- } \beta \text {-araf- }(1->2)-5-0 \text { - } \\
\text { mycolyl- } \alpha \text {-araf- }\left(1->1^{\prime}\right) \text {-glycerol }\end{array}$ & TIMP3 & IFN- $\alpha$ & Fenretinide \\
\hline Leukotriene D4 & TO-901317 & Poly rl:rC-RNA & miR-124-39 (and other miRNAs with seed AAGGCAC) \\
\hline Kainic acid & Rottlerin & PRL & STK11 \\
\hline TLR4 & Nr1h & IFN- $\beta$ & Mir-21 \\
\hline TLR9 & DUSP1 & STAT1 & GAPDH \\
\hline TLR & NPPA & IRF3 & Irgm1 \\
\hline SELPLG & Cyclosporin A & IFN & Curcumin \\
\hline \multirow[t]{4}{*}{ Phorbol myristate acetate } & n-3 fatty acids & & \\
\hline & TNFAIP3 & & \\
\hline & APLN & & \\
\hline & Superoxide & & \\
\hline
\end{tabular}

Analysis was performed based on upregulated/downregulated genes between groups indicated in the second row.

Pathway analysis demonstrates differential immunologic response patterns between $S D, P R$, and CR patients. We next performed ingenuity pathway analysis using IPA software (Qiagen). We selected the top one thousand most statistically different genes in each group at the week $0,6,15$, and 34 time points for analysis at each time point (Figure 3 and Supplemental Figure 3). At week 0, patients with PR, but not patients with $\mathrm{CR}$, demonstrated increased LXR/RXR activation (regulation of lipid metabolism, inflammation, and cholesterol to bile acid catabolism), DC maturation, Th1 pathway and IFN signaling, and decreased TREM1 signaling (monocytic chemokine regulation) compared with SD patients (Supplemental Figure 3A). Although, according to IPA analysis, patients with PR demonstrated a reduction of the "role of pattern recognition receptors (PRR) in recognition of bacteria and viruses" pathway (PRR pathway) compared with SD patients, this did not include differential expression of $T L R 3$, encoding a receptor for Poly-IC:LC. At week 6, the most significantly enriched pathway in SD patients compared with CR or PR patients related to chemokine pathway signaling (Supplemental Figure 3B). Additionally, although PR patients had overall decreased expression of genes in the PRR pathways, TLR3 expression was increased in these patients compared with SD patients. At week 15, IFN signaling was the most significantly enriched pathway in both PR and CR patients compared with SD patients (Supplemental Figure 3C). However, CR patients generally showed decreased expression of genes in the Th2 pathway, while PR patients had increased chemokine pathway (TREM1) expression levels compared with SD patients. Finally, by week 34, CR patients, but not PR patients, demonstrated strikingly elevated expression of genes in the IFN, Th1, and PRR pathways (Figure 3). Notably, at week 34, both PR and CR patients demonstrated enriched TREM1 signaling compared with SD patients.

We next assessed predicted upstream regulators of our gene pathways at each time point using IPA software (Table 2). Remarkably, at week 34 after initiation of treatment, CR patients demonstrated IFN and Poly-IC adjuvant responses, while patients with PR only demonstrated Poly-IC responses but not IFN signaling responses and patients with SD demonstrated neither a strong Poly-IC nor a IFN response. Of note, patients with CR showed decreased expression of the COX-2/PGE2 pathway at weeks 0 (salicylic acid) and 15 (aspirin) (Supplemental Table 1). 
Low GSTM1 expression is associated with CR compared with SD and PR. We next examined genes differentially expressed between $C R$ and PR patients. Our differential expression analysis revealed that, at every time point analyzed, patients with $\mathrm{CR}$ demonstrated significantly lower expression of the gene coding for glutathione- $S$-transferase $\mu 1$ (GSTM1) compared with PR patients (Figure 4). Notably, SD patients demonstrated similar GSTM1 expression levels to those of PR patients (Figure 4B). Additionally, we observed that, at 6 weeks after initiation of treatment, patients with CR demonstrated significantly elevated T cell cytotoxic molecules, $P R F 1$ and $G Z M B$, compared with SD patients (Figure 4A). Thus, in our patient cohort, GSTM1 and early cytotoxic T cell genes predicted patients with CR To test whether GSTM1 might predict outcomes outside the context of immunotherapy, we performed survival analysis on public data (http://gliovis.bioinfo.cnio.es/) of adult and pediatric tumors from nonvaccinated patients (Supplemental Figure 4). GSTM1 was not associated with outcomes in any of the assessed databases.

Survivin ELISPOT counts correlate with $T$ cell activation pathways. We previously reported that all patients in this study demonstrated striking biologic responses (ELISPOT) compared with our pediatric high-grade glioma and adult vaccine-treated patients and that ELISPOT responses were associated with radiologic responses $(2-4,14)$. To identify peripheral immune genes and pathways associated with ELISPOT responses, we performed Pearson correlation coefficient (PCC) analysis to assess expression values correlating with ELISPOT counts. IPA of significantly correlating genes $(n=1,000, P<$ 0.05 ) identified pathways related to DC activation of $\mathrm{T}$ cells and Th1 cells, which positively correlated with survivin ELISPOT counts (Figure 5A and Supplemental Figure 5). Surprisingly, few immunologic pathways correlated with both IL-13Ra2 and EphA2 ELISPOT counts (Supplemental Figure 5, B and $\mathrm{C}$, respectively). We additionally performed a pathway comparison analysis to identify pathways with similar overall expression between each ELISPOT condition (Figure 5B; IL-13Ra2, survivin, and EphA2). Although we observed significant discordance among pathways correlating with each peptide ELISPOT response, we did observe several concordant pathways that positively and inversely correlated with ELISPOT responses to all 3 peptides (Figure 5B). Our correlation analysis of gene expression signatures with ELISPOT counts, irrespective of clinical course, suggests that specific expression patterns, at least in part, are likely the result of specific immune responses and do not only reflect the clinical course. However, further studies are warranted to establish peripheral immune responses associated with clinical course in these patients.

Finally, we were interested in finding markers that were correlated with PFS, especially at early time points, as these could serve as biomarkers of treatment response. Interestingly, high levels of $\mathrm{T}$ cell markers $C D 3 \mathrm{D}$ and $C D 4$ prior to treatment were positively correlated with longer PFS. This confirms our previous observation of reduction in memory $\mathrm{T}$ cell gene sets in SD patient PBMCs compared with CR patient PBMCs (Supplemental Figure 2B). Additionally, we found a negative correlation of CD274 (PD-L1) and IDO1 levels, both encoding checkpoint proteins, with PFS at week 0 (Figure 5C). Along with the aforementioned upregulation of $\mathrm{T}$ cell cytotoxic molecules, $P R F 1$ and GZMB, in PBMC samples from CR patients after 6 weeks of treatment, we observed a strong correlation of expression of $\mathrm{T}$ cell activation markers CD26, CD38, CD69, and CD40LG with PFS at week 6.

\section{Discussion}

Our recent pilot trial of peptide vaccine immunotherapy, involving 3 immunogenic protein epitopes, demonstrated CR responses in 2 of 12 children with recurrent LGGs and PR responses in 3 others (14). Furthermore, we previously reported on the robust immunologic responses by ELISPOT, in all assessed LGG patients (14). These were significantly higher compared with our previous experiences with both pediatric and adult high-grade gliomas $(2,3)$, which likely relates to the unique characteristics of LGGs. The promising clinical and biologic responses in these patients led us to initiate a phase II clinical trial using this peptide vaccine strategy (NCT02358187).

While the results of work to date are highly encouraging, our pilot trial also demonstrates a clear need for developing approaches to predict which patients will respond to or resist treatment. A lack of any patients with CR likely reflects issues of immune escape, lack of antigen processing components, the development of an unfavorable immune milieu, or upregulation of immune checkpoint molecules. Additionally, the variability in responses prompted us to assess biomarkers of response and resistance to our therapy. 

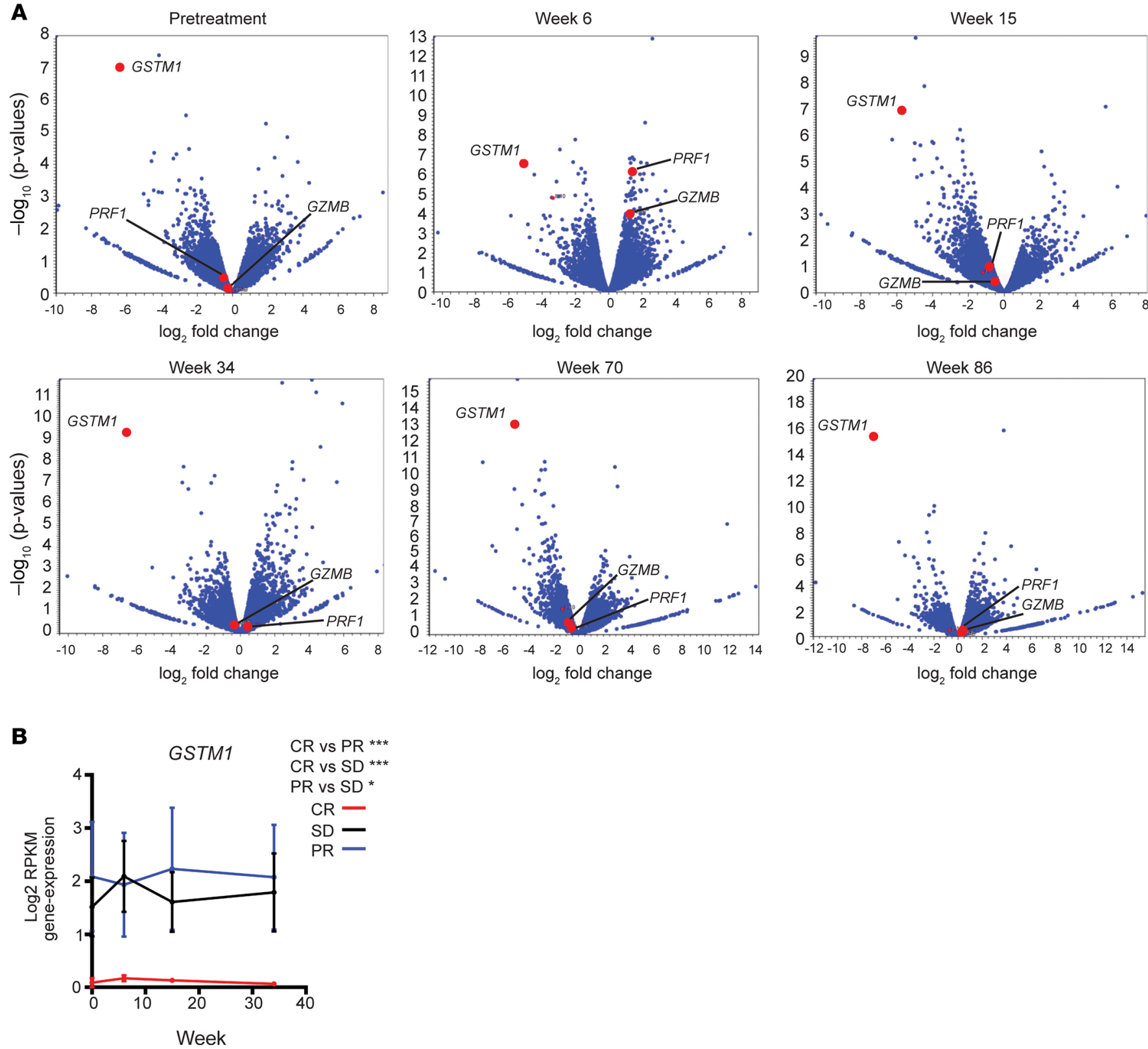

Figure 4. Differential expression analysis between $C R$ and PR patients. (A) Volcano plots comparing fold change ( $x$ axis) and $P$ value ( $y$ axis, EdgeR) for 36,410 expressed genes at $0,6,15,34,70$, and 86 weeks between CR ( $n=2$ patients) and PR $(n=3)$. Specific genes are highlighted in red. (B) Average ( \pm SD) expression of GSTM1 (y axis) from week 0 to 34 ( $x$ axis). Response groups are indicated by color. ${ }^{* *}$ Adjusted $P<0.0001$ (Tukey's test with multiple testing correction). ${ }^{*}$ Adjusted $P<0.05$ (Tukey's test).

Our findings demonstrate significantly elevated expression of $H L A-V$, an $\mathrm{MHC}$ class I pseudogene, in $\mathrm{SD}$ patients both prior to treatment and at every time point assessed. Peptide vaccines are critically dependent on HLA class I expression. Although we do not yet know if $H L A-V$ biologically interferes with vaccine responses, pseudogenes have been shown to exert a dominant negative effect on other genes (reviewed in ref. 22). Thus, additional studies are warranted to better understand the biologic consequences of $H L A-V$ expression. Interestingly, $H L A-V$ remained elevated at every time point in SD patients compared with $\mathrm{PR}$ and $\mathrm{CR}$ patients. In the current study, we assessed both coding and noncoding mRNAs, which allowed us to detect the $H L A-V$ pseudogene. As such, previous studies examining only coding RNAs may have failed to identify $H L A-V$. Our data suggest that future studies should include the analysis of coding and noncoding RNAs. Although our study was limited to peptide vaccines, which have a unique requirement for peptide binding to HLA molecules, it is possible that any therapy involving class I-restricted antigen spreading may demonstrate similar biomarkers. 
A Positive z-score $\square$ Negative z-score
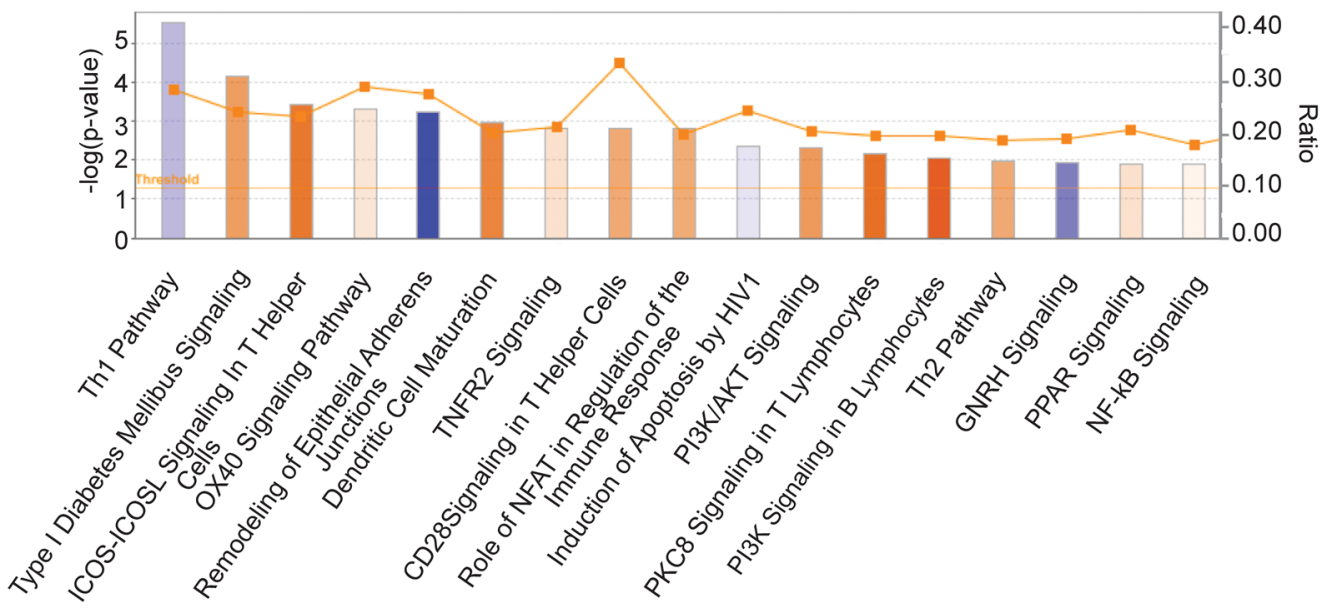

B Activation z-score

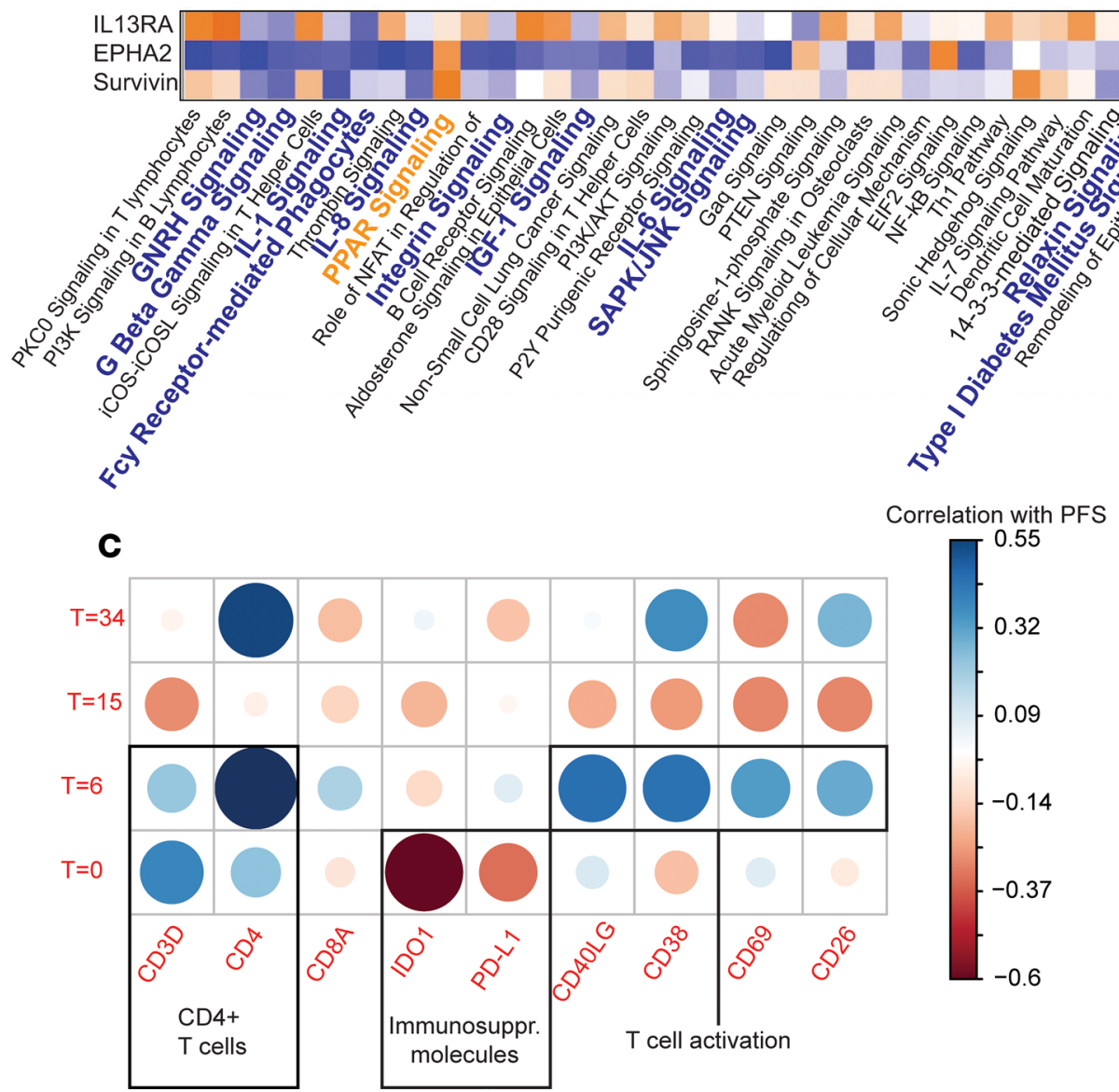

Figure 5. Expression of immune-specific pathways correlates with survivin ELISPOT counts. (A) Significance (y axis, Fisher's exact test) of pathway enrichment for genes significantly correlating with ELISPOT counts. The ratio of significantly correlated genes to the total number of genes in the pathway is shown by the orange line. The average correlation Z-score of genes in each pathway is indicated by color. (B) Average activation Z-score (color) for pathways (columns) across 3 ELISPOTs (rows). Pathways with concordant activation score are highlighted. (C) Correlation of expression of indicated genes (columns) at different time points (rows) with PFS across all patients. 
Monocyte-related genes were among the most upregulated genes expressed in SD patients at the earliest time point assessed (week 6) and at a later time point (week 15) in PR patients compared with CR patients. This finding is consistent with our previous work, which demonstrated in multiple models that tumor-infiltrating myeloid cells can reduce $\mathrm{T}$ cell activity and promote tumor growth $(9,13,23)$. Further studies are warranted to examine the role of peripheral blood monocyte pathways in immunosuppression in gliomas.

We identified lower IDO1 expression levels in patients with PFS $>20$ weeks compared with patients with PFS $<20$ weeks. IDO1 is part of the process in which tryptophan (Trp) is converted into kynurenine (Kyn). This pathway plays a key role in driving immunosuppression in many cancers and is consequently a target for therapy (24). It has previously been shown that there is a trend of higher Kyn/Trp levels in GBM patients with decreased survival (25). Furthermore, nontumor-derived IDO1 possesses most of the Trp catabolic activity in mice injected with GL261. This analysis incorporated cervical lymph nodes in WT and IDO1-knockout mice, implicating peripheral IDO1 in modulating the antitumor immune response (26). Our finding that low IDO1 levels in peripheral blood before vaccination (week 0) were strongly correlated with longer PFS in our cohort suggests that SD patients may benefit from additional checkpoint blockade approaches, such as IDO inhibitors.

Members of the polymorphic family of glutathione-S-transferases (GSTs) are phase 2 enzymes that contribute to xenobiotic detoxification (27). Chemotherapeutics, including alkylating agents and free radicals produced by radiation, belong to this category of xenobiotic agents. As a consequence, studies have demonstrated overexpression of GSTM1 in chemotherapy-resistant malignant cell lines (28). Our data suggest that GSTM1 deficiency may also predict response to vaccination in children with recurrent LGG. Homozygous deletions of the locus encoding GSTM1, which results in complete lack of the GSTM1 protein, is observed in $40 \%-50 \%$ of the population (29). Interestingly, in patients with anaplastic astrocytoma as well as oligodendroglioma, the GSTM1-null genotype, in combination with a specific homozygous GSTM1 allelic variant, infers superior survival and increased chemotherapy-related toxicity (30). More recent studies have associated GSTM1 mRNA levels with type 1 Th cell differentiation in aging (31), suggesting that GSTM1 deficiency may effect CD4 T cell function. The exact mechanism of GSTM1 and if GSTM1 deletion is a predictor of vaccination response in children with recurrent LGG remain to be elucidated in a larger cohort.

Pretreatment biomarkers may be ideal for distinguishing which patients to treat, from the onset. Prospective studies are warranted to determine whether GSTM1 and HLA-V predict patient outcome following peptide vaccine immunotherapy. Notably, peripheral immune pathway correlation analysis was a described secondary endpoint for the presented vaccine trial. Accordingly, samples and dates were carefully saved for this analysis. Moreover, correlation analysis of gene expression signatures with ELISPOT counts, irrespective of clinical course, suggests that specific expression patterns, at least in part, are likely attributed to specific immune responses and do not only reflect the clinical course. However, further studies are warranted to establish peripheral immune responses associated with clinical course in these patients.

Immunomonitoring at a cellular level in cancer immunotherapy trials is technically challenging, difficult to standardize, and often poorly correlative with clinical outcome. Development of gene expression analyses, such as those used here, could be very important for oncoimmunology trial development. A clear limitation of our study is the extremely small sample population. It is very difficult to draw firm conclusions based on a very small number of responders, taking known interpatient variation parameters into account. In view of the very small size of the patient group and the heterogeneous radiographic responses, it remains speculative as to how many of the identified gene signatures will ultimately be validated in prospective randomized trials. As we have started enrolling patients in a similar phase II trial, we plan to further evaluate these and other genes in our current trial with greater numbers of patients. In addition to the small sample population size, we acknowledge another limitation of our study intrinsic to using PBMC expression profiles: these profiles are highly variable and subject to a multitude of influences, such as medication, diet, infections, etc. Furthermore, potential changes in expression patterns may be diluted. Nevertheless, in our study we found that genes, such as GSTM-1 and $H L A-V$, were upregulated in SD patients at every time point assessed, suggesting that these genes are less variable due to external factors compared with other genes. Future studies should consider performing analysis on multiple cell populations separately, such as tetramer ${ }^{+} \mathrm{T}$ cells. Despite these limitations, our findings demonstrate that immune patterns may be identified in PBMCs by RNA-seq analysis. Thus, RNA-seq of peripheral samples represents a potentially useful immune-monitoring tool for use in future immuno-oncology trials. 
We summarize our findings in Supplemental Figure 6. Overall, our data illustrate for the first time to our knowledge that peripheral biomarkers and immune response patterns may be associated with response to peptide vaccine immunotherapy treatment in pediatric LGG patients. We observed in our cohort that GSMT1 and HLA-V expression was associated with patient responses both before treatment and at every time point following peptide vaccine immunotherapy. Additionally, patients with $C R$ demonstrated week 6 induction of cytotoxic T cell markers, GZMB and PRF1, and a robust IFN and Poly-IC response at 34 weeks compared with SD and PR patients. Conversely, patients with PR experienced increased expression of monocytic pathways at week 15 and a Poly-IC, but not IFN, response at week 34. Patients with SD experienced an early (week 6) monocytic hematopoietic response.

\section{Methods}

PBMC isolation. PBMCs were isolated from whole blood using Ficoll, according to the manufacturer's protocol (GE Healthcare), at the Immunologic Monitoring and Cellular Products Laboratory at the Hillman Cancer Center.

RNA isolation and library prep. DNase-1-treated RNA was isolated using a Direct-zol RNA isolation kit (Zymo). Each sample was assessed using a Qubit 2.0 fluorometer and Agilent 4 for RNA quantity and quality. All RNA integrity numbers (RIN) exceeded RIN-8. Total RNA libraries were generated using the Illumina TruSeq Stranded mRNA (Poly-A+) sample preparation kit. The first step in the workflow was purifying the Poly-A-containing mRNA molecules using Poly-T oligo-attached magnetic beads. Following purification, the mRNA was fragmented into small pieces using divalent cations. The cleaved RNA fragments were copied into first-strand cDNA using reverse transcriptase and random primers. Strand specificity was achieved by using dUTP in the Second Strand Marking Mix, followed by second-strand cDNA synthesis using DNA Polymerase I and RNase H (all obtained from Illumina). These cDNA fragments then had the addition of a single " $\mathrm{A}$ " base and subsequent ligation of the adapter. The products were then purified and enriched with PCR to create the final cDNA library.

The cDNA libraries were validated using the KAPA Biosystems primer premix kit with Illumina-compatible DNA primers and a Qubit 2.0 fluorometer. Quality was examined using Agilent TapeStation 2200. The cDNA libraries were pooled at a final concentration of $1.8 \mathrm{pM}$. Cluster generation and 75-bp pairedread dual-indexed sequencing was performed on Illumina NextSeq 500's using a high-output 150 cycle kit (Illumina). Approximately 40 million paired reads were collected for each sample.

RNA-seq analysis. Quality control, sequence alignment, expression quantification, and Volcano plot generation were performed using CLC Genomics Workbench (Qiagen). In the mapping step, human hg38 was used as reference genome, and the ENSEMBL version $81 \mathrm{gtf}$ track was used to quantify gene expression. Only correctly paired, uniquely mapped reads were considered. For each sample, expression values were scaled to TPM. PCA plots were generated in $\log 2$ count space with the R scater package (32), and consensus clustering was carried out with the SC3 R package, where k was set to 2 (33). Clustering results were visualized with Morpheus from the Broad Institute (https://software.broadinstitute.org/morpheus/). Differential expression analysis was calculated in the CLC Genomics Workbench, which implements the "Empirical analysis of DGE" algorithm, using for the most part the default settings in the edgeR package (version 3.4.0). Deconvolution of PBMC samples was performed with xCell via its web interface (http://xcell.ucsf.edu/). TPM-scaled data were submitted to the web service, and relative estimates for 64 immune and stroma cell types were returned online. These were used to compare estimates between groups at different time points.

GSEA was performed as follows: to identify pathways that were associated with response to our vaccine, we compared RNA-seq data from CR, PD, and SD patients at week 0 . A Wilcoxon rank-sum test was performed on $\log 2(\mathrm{TPM}+1)$ transformed data. Significance was established as genes differentially expressed with a minimum of 1.5 -fold change and a $P<0.05$ in R. Significant genes were then inputted as a preranked list into GSEA 3.0 (build: 0160) with Hallmark Immune Gene Signature (v6.1) used to identify significantly enriched immune pathways.

IPA analysis. Data were uploaded into IPA, and core analysis was performed. Expression log ratios were used to calculate $Z$-score values. We focused on both upregulated and downregulated genes using the Ingenuity Knowledge bases (genes) as a reference set. A score cutoff of $-\log (P$ value $)$ greater than 1.3 and an absolute value $Z$-score of 0.1 were used. Scoring was performed using Fisher's exact test $P$ values.

Correlation analysis. To determine genes associated with PFS, we calculated the PCC for each gene and the PFS time across all 12 patients using R 3.4.1. To determine genes associated with ELISPOT counts, 
we calculated the PCC for each gene with respect to IL-13RA, EphA2, and survivin ELISPOT data across all 12 patients and time points in $\mathrm{R}$ 3.4.1. Correlations of $\mathrm{r}> \pm 0.4$ with a $Z$-score of $> \pm 2.0(P<0.05)$ were considered significant.

Survival analysis. Survival analysis was carried out with the Gliovis portal (http://gliovis.bioinfo. cnio.es/) (34).

Availability of data and material. The data sets supporting the conclusions of this article are available in the ArrayExpress repository, under accession E-MTAB-6270 (https://www.ebi.ac.uk/arrayexpress/).

Statistics. Differential expression analysis was performed as implemented in the CLC Genomics Workbench (version 10.1.1), which utilizes the edgeR package (version 3.4.0). Pathway enrichments (Fisher's exact test) and activation scores (see http://pages.ingenuity.com/rs/ingenuity/images/0812\%20 upstream_regulator_analysis_whitepaper.pdf) were calculated via the IPA software (version 10.1.1). All other analyses were carried out in R (version 3.4.1). Two-tailed $t$ tests were used. $P$ values of less than 0.05 were considered significant.

Study approval. All experiments were carried out in conformity with the principles set out in the World Medical Association's Declaration of Helsinki as well as the Department of Health and Human Services Belmont Report. The University of Pittsburgh Institutional Review Board approved sample use (PRO08030085). Informed written consent was provided by all patients prior to inclusion in the study.

\section{Author contributions}

GK, SA, LHB, HO, and IFP conceived of and designed the study. GK performed the RNA isolation. GK, SA, SM, MIM, NS, AC, AVL, SC, KES, CRV, and AD performed the bioinformatics analysis. GK and SM wrote the manuscript, with input from all the authors. All authors read and approved the final manuscript.

\section{Acknowledgments}

We thank William Horne and Annabel Ferguson at the Health Sciences Sequencing Core at Children's Hospital of Pittsburgh for assistance with RNA-seq; University of Pittsburgh Cancer Institute Clinical Research Services for regulatory management; Andres Salazar, Oncovir Inc., for provision of Poly-IC:LC; and Lauren H. McCarl for editing the manuscript. This study was supported by the St. Baldrick's Foundation; the Henry Cermak Fund for Pediatric Cancer Research, a St. Baldrick's Hero Fund; the Immunologic Monitoring and Cellular Products Laboratory at the Hillman Cancer Center; the Pediatric Clinical and Translational Research Center; and NIH grants R01CA187219, UL1 RR024153, UL1TR000005, and P30CA47904. Support was also provided by grants from the Pediatric Low-Grade Glioma Initiative via the National Brain Tumor Society, Ellie Kavalieros Fund, Connor's Cure Fund, Ian Yagoda's Friends Foundation, and the Translational Brain Tumor Fund of the Children's Hospital of Pittsburgh Foundation.

Address correspondence to: Gary Kohanbash, Department of Neurological Surgery, University of Pittsburgh, Children's Hospital of Pittsburgh of UPMC, 7131 Rangos Research Building, 530 45th Street, Pittsburgh, Pennsylvania 15201, USA. Phone: 412.692.9456; Email: gary.kohanbash2@chp.edu.

1. Okada $\mathrm{H}$, et al. Induction of robust type-I CD8+ T-cell responses in WHO grade 2 low-grade glioma patients receiving peptide-based vaccines in combination with poly-ICLC. Clin Cancer Res. 2015;21(2):286-294.

2. Okada H, et al. Induction of CD8+ T-cell responses against novel glioma-associated antigen peptides and clinical activity by vaccinations with \{alpha\}-type 1 polarized dendritic cells and polyinosinic-polycytidylic acid stabilized by lysine and carboxymethylcellulose in patients with recurrent malignant glioma. J Clin Oncol. 2011;29(3):330-336.

3. Pollack IF, et al. Antigen-specific immunoreactivity and clinical outcome following vaccination with glioma-associated antigen peptides in children with recurrent high-grade gliomas: results of a pilot study. J Neurooncol. 2016;130(3):517-527.

4. Pollack IF, et al. Antigen-specific immune responses and clinical outcome after vaccination with glioma-associated antigen peptides and polyinosinic-polycytidylic acid stabilized by lysine and carboxymethylcellulose in children with newly diagnosed malignant brainstem and nonbrainstem gliomas. J Clin Oncol. 2014;32(19):2050-2058.

5. Yu JS, Liu G, Ying H, Yong WH, Black KL, Wheeler CJ. Vaccination with tumor lysate-pulsed dendritic cells elicits antigen-specific, cytotoxic T-cells in patients with malignant glioma. Cancer Res. 2004;64(14):4973-4979.

6. Sampson JH, et al. Immunologic escape after prolonged progression-free survival with epidermal growth factor receptor variant III peptide vaccination in patients with newly diagnosed glioblastoma. J Clin Oncol. 2010;28(31):4722-4729.

7. Wu A, et al. Expression of MHC I and NK ligands on human CD133+ glioma cells: possible targets of immunotherapy. J Neurooncol. 2007;83(2):121-131.

8. Xue S, Song G, Yu J. The prognostic significance of PD-L1 expression in patients with glioma: A meta-analysis. Sci Rep. 2017;7(1):4231. 
9. Kohanbash G, Okada H. Myeloid-derived suppressor cells (MDSCs) in gliomas and glioma-development. Immunol Invest. 2012;41(6-7):658-679.

10. Sasaki K, et al. IL-4 suppresses very late antigen-4 expression which is required for therapeutic Th1 T-cell trafficking into tumors. J Immunother. 2009;32(8):793-802.

11. Sasaki K, et al. Preferential expression of very late antigen-4 on type 1 CTL cells plays a critical role in trafficking into central nervous system tumors. Cancer Res. 2007;67(13):6451-6458.

12. Müller S, et al. Single-cell profiling of human gliomas reveals macrophage ontogeny as a basis for regional differences in macrophage activation in the tumor microenvironment. Genome Biol. 2017;18(1):234.

13. Fujita M, et al. COX-2 blockade suppresses gliomagenesis by inhibiting myeloid-derived suppressor cells. Cancer Res. 2011;71(7):2664-2674

14. Pollack IF, et al. Immune responses and outcome after vaccination with glioma-associated antigen peptides and poly-ICLC in a pilot study for pediatric recurrent low-grade gliomas. Neuro-oncology. 2016;18(8):1157-1168.

15. Shoemaker JE, Lopes TJ, Ghosh S, Matsuoka Y, Kawaoka Y, Kitano H. CTen: a web-based platform for identifying enriched cell types from heterogeneous microarray data. BMC Genomics. 2012;13:460

16. Monti S, Tamayo P, Mesirov J, Golub T. Consensus Clustering: A resampling-based method for class discovery and visualization of gene expression microarray data. Mach Learn. 2003;52(1):91-118.

17. Robinson MD, McCarthy DJ, Smyth GK. edgeR: a Bioconductor package for differential expression analysis of digital gene expression data. Bioinformatics. 2010;26(1):139-140.

18. Aran D, Hu Z, Butte AJ. xCell: digitally portraying the tissue cellular heterogeneity landscape. Genome Biol. 2017;18(1):220.

19. Sun Y, Cai J, Ma F, Lü P, Huang H, Zhou J. miR-155 mediates suppressive effect of progesterone on TLR3, TLR4-triggered immune response. Immunol Lett. 2012;146(1-2):25-30.

20. Hu X, Ye J, Qin A, Zou H, Shao H, Qian K. Both microRNA-155 and virus-encoded miR-155 ortholog regulate TLR3 expression. PLoS ONE. 2015;10(5):e0126012.

21. Dudda JC, et al. MicroRNA-155 is required for effector CD8+ T cell responses to virus infection and cancer. Immunity. 2013;38(4):742-753.

22. Milligan MJ, Lipovich L. Pseudogene-derived lncRNAs: emerging regulators of gene expression. Front Genet. $2014 ; 5: 476$.

23. Kohanbash G, et al. GM-CSF promotes the immunosuppressive activity of glioma-infiltrating myeloid cells through interleukin-4 receptor- $\alpha$. Cancer Res. 2013;73(21):6413-6423.

24. Zhai L, et al. The role of IDO in brain tumor immunotherapy. J Neurooncol. 2015;123(3):395-403.

25. Zhai L, et al. The kynurenine to tryptophan ratio as a prognostic tool for glioblastoma patients enrolling in immunotherapy. $J$ Clin Neurosci. 2015;22(12):1964-1968.

26. Zhai L, et al. Non-tumor cell IDO1 predominantly contributes to enzyme activity and response to CTLA-4/PD-L1 inhibition in mouse glioblastoma. Brain Behav Immun. 2017;62:24-29.

27. Kilburn L, et al. Glutathione S-transferase polymorphisms are associated with survival in anaplastic glioma patients. Cancer. 2010;116(9):2242-2249.

28. Morgan RA, et al. Cancer regression and neurological toxicity following anti-MAGE-A3 TCR gene therapy. J Immunother. 2013;36(2):133-151.

29. Harrison DJ, Cantlay AM, Rae F, Lamb D, Smith CA. Frequency of glutathione S-transferase M1 deletion in smokers with emphysema and lung cancer. Hum Exp Toxicol. 1997;16(7):356-360.

30. Okcu MF, et al. Glutathione S-transferase polymorphisms and survival in primary malignant glioma. Clin Cancer Res. 2004;10(8):2618-2625

31. Yeh SH, Liu CL, Chang RC, Wu CC, Lin CH, Yang KD. Aging-dependent DNA hypermethylation and gene expression of GSTM1 involved in T cell differentiation. Oncotarget. 2017;8(30):48591-48602.

32. McCarthy DJ, Campbell KR, Lun AT, Wills QF. Scater: pre-processing, quality control, normalization and visualization of single-cell RNA-seq data in R. Bioinformatics. 2017;33(8):1179-1186.

33. Kiselev VY, et al. SC3: consensus clustering of single-cell RNA-seq data. Nat Methods. 2017;14(5):483-486.

34. Bowman RL, Wang Q, Carro A, Verhaak RG, Squatrito M. GlioVis data portal for visualization and analysis of brain tumor expression datasets. Neuro-oncology. 2017;19(1):139-141. 